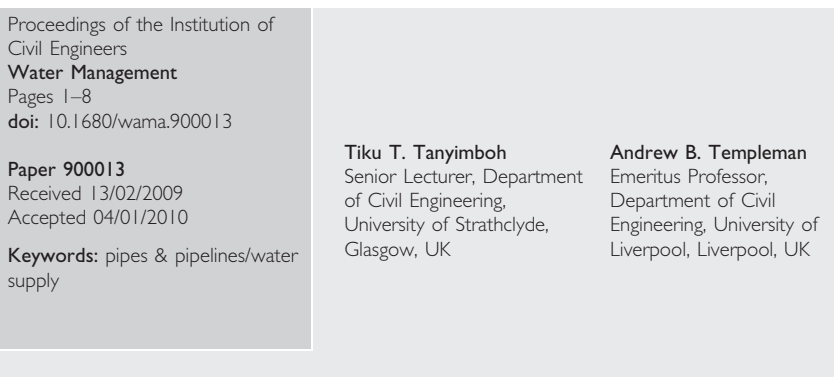

\title{
Seamless pressure-deficient water distribution system model
}

T. T. Tanyimboh PhD and A. B. Templeman PhD

Pressure-deficient conditions are a common occurrence in water distribution systems. These situations require accurate modelling for timely decision making. However, the conventional demand-driven analysis approach to network modelling is unsuitable for operating conditions with insufficient pressure. Increasing emphasis is being placed on the need for water companies to satisfy stringent performance standards for the continuous supply of water to consumers and it is those pressuredeficient operating conditions which are critical in determining whether or not adequate supplies can be provided. It is therefore very unfortunate that the demand-driven analysis method becomes invalid for use in precisely those critical conditions. The aim of this paper is to present a new pressure-dependent demand function to help improve the simulation of pressuredeficient conditions. The proposed function has better computational properties than those in the literature and has been incorporated successfully in the governing equations for water distribution networks. In particular, the proposed function and its derivative do not have the discontinuities that often cause convergence difficulties in the solution of the constitutive equations. A robust Newton-Raphson algorithm was developed to model water distribution systems under both normal and pressure-deficient conditions in a seamless way. Examples which demonstrate the methodology are included.

\section{NOTATION}

$C_{i j} \quad$ Hazen-Williams roughness coefficient of pipe $i j$

c a coefficient in the head-discharge relationship for a pump

$D_{i j} \quad$ diameter of pipe $i j$

$E_{j} \quad$ elevation of node $j$

$F_{j} \quad$ flow balance (i.e. continuity equation) for node $j$

$H_{j} \quad$ piezometric head at node $j$

$H_{j}^{\text {des }} \quad$ piezometric head at node $j$ above which its demand is satisfied in full

$H_{j}^{\text {min }} \quad$ piezometric head at node $j$ below which its flow is zero

$H_{\text {psv }} \quad$ setting for pressure sustaining valve

$H_{\text {set }} \quad$ valve setting

$h_{0} \quad$ shut-off head for pump

$h_{\mathrm{p}} \quad$ head added by pump

$J \quad$ Jacobean matrix

$L_{i j} \quad$ length of pipe $i j$ $m$

a coefficient in the head-discharge relationship for a pump

$N_{i} \quad$ the set of nodes connected to node $i$

$\boldsymbol{Q}_{i j} \quad$ volume flow rate through link $i j$

$\boldsymbol{Q}_{j} \quad$ flow at node $j$ (same as $\boldsymbol{Q}_{j}^{\text {avl }}$ )

$Q_{j}^{\text {avl }} \quad$ flow at node $j$

$\boldsymbol{Q}_{j}^{\mathrm{req}} \quad$ demand at node $j$

$\boldsymbol{Q}_{\mathrm{p}} \quad$ volume flow rate through a pump

$\alpha_{j} \quad$ a parameter in the proposed pressure-dependent demand function

$\beta_{j} \quad$ a parameter in the proposed pressure-dependent demand function

$\delta \quad$ dimensionless factor in the headloss formula

$\varepsilon \quad$ a tolerance parameter in the convergence criteria

$\lambda$ relaxation coefficient in the Newton-Raphson algorithm

$\mu_{j} \quad$ mean value of $H_{j}-E_{j}$

$\sigma_{j} \quad$ standard deviation of $H_{j}-E_{j}$

\section{INTRODUCTION}

Pressure-deficient conditions are a common occurrence in water distribution systems (WDSs). Examples include pump failures, pipe bursts, excessive demands (e.g. for fire-fighting purposes) and when major pipes are taken out of service in order to carry out maintenance and repairs. These situations require accurate simulation of the WDS for timely decision making. However, the conventional demand-driven analysis (DDA) approach to network modelling is unsuitable for operating conditions with insufficient pressure (indicated by large negative pressures at some demand nodes, for example). Head-driven analysis (HDA) is much closer to reality because it takes account of the pressures at the demand nodes.

Recent reviews of head-dependent modelling include Ackley et al. (2001), Tabesh et al. (2002) and Tanyimboh et al. (2003). Bhave (1991) categorised demand nodes as fully satisfactory if the piezometric head was not less than the head required, or no-flow if the head was below the elevation of the node. All other nodes were classed as partial-flow. Each subnormal node was modelled as a ground-level tank to determine the flow available. The procedure is very laborious and time-consuming, impracticable on real WDSs and does not lend itself to a range of analyses, such as reliability and optimisation studies. Tanyimboh et al. (1999) implemented a more 
practicable version of the technique. The algorithm proposed by Ang and Jowitt (2006) uses artificial reservoirs in a similar manner to Bhave (1991). Rossman (2007) formalised the procedure using emitters in a methodology that involves a single DDA run. Gupta and Bhave (1996) used multiple DDA runs to adjust nodal flows iteratively. Chandapillai (1991) updated nodal flows between successive DDA solutions but the individual demand nodes were updated in isolation thus causing convergence problems.

Kalungi and Tanyimboh (2003) developed a heuristic in which some aspects of HDA were used in a DDA environment to yield more realistic results. Some features of the technique include the iterative use of DDA, a subcategory of nodes referred to as key partial flow nodes and a joint nodal head-and-nodal flow system of equations. The method does not make explicit use of a pressure-dependent demand function (PDDF). A major advantage of the methods in which the PDDFs are embedded in the system of equations is that they solve the non-linear constitutive equations only once, albeit iteratively. Tabesh et al. (2002) used a relaxation coefficient whose value is obtained by trial and error. Ackley et al. (2001) used a mathematical programming formulation in which the objective was to maximise the amount of water delivered while satisfying the system constraints. Recently Giustolisi et al. (2008) and Siew and Tanyimboh (2009) added PDDFs to the gradient method (Todini and Pilati, 1988); pumps and valves were not catered for.

This paper presents a new PDDF that is based on the logit function (Weisberg, 1985). The proposed function has better computational properties than those in the literature and has been incorporated successfully in the system of equations for WDSs. A robust Newton-Raphson algorithm was developed to model WDSs under both normal and pressure-deficient conditions in a seamless way. The formulation in Tanyimboh et al. (2003) has been extended to other components including pumps and valves. Examples that demonstrate the methodology are included.

\section{PRESSURE-DEPENDENT DEMAND FUNCTIONS}

There is general agreement that the flow rate at a demand node is related to the local pressure in the WDS. There is, however, some uncertainty about the exact nature of the relationship between flow and pressure due to a range of factors. The relevant considerations may include the proximity of other abstractions and their timings and magnitudes, elevations of demand nodes, supply pipe characteristics, internal plumbing and storage arrangements, and the micro-component make-up of demands. It may be noted that water demand is inherently stochastic which, therefore, introduces an extra level of uncertainty.

Several functions have been proposed to characterise the relationship between the nodal pressures and flows in WDSs (Cullinane et al., 1992; Fujiwara and Ganesharajah, 1993; Gupta and Bhave, 1996; Reddy and Elango, 1989; Wagner et al., 1988). These PDDFs have been defined on the basis that the nodal demand is fully satisfied when the nodal piezometric head is greater than the desired level and zero when the piezometric head is below the minimum level. A selection of PDDFs from the literature are summarised in Table 1. Some weaknesses of previous PDDFs include the absence of continuity in their derivatives at the transitions between zero and partial nodal flow and/or between partial and full demand satisfaction. Discontinuities in PDDFs and their derivatives can cause convergence difficulties in the computational solution of the system of equations for WDSs (Gupta et al., 2003).

Furthermore, some PDDFs can yield demand satisfaction ratios (DSRs) that either exceed, or never reach, 100\%

(Germanopoulos, 1985; Reddy and Elango, 1989). Similarly, others give nodal flows that are significantly greater than zero when the residual pressure is zero (Cullinane et al., 1992).

Gupta and Bhave (1996) recommended Equation 1 (Wagner et al., 1988). They also proposed Equation 2 as an improvement on Germanopoulos (1985). Udo and Ozawa (2001) observed that the Wagner et al. (1988) square root-type PDDF is similar to the characteristic curve of a valve and appropriate for

\begin{tabular}{|c|c|c|c|}
\hline & $\begin{array}{l}\text { Zero flow } \\
\left(H_{j} \leqslant H_{j}^{\text {min }}\right)\end{array}$ & $\begin{array}{l}\text { Partial flow } \\
\left(H_{j}^{\text {min }}<H_{j}<H_{j}^{\text {des }}\right)\end{array}$ & $\begin{array}{l}\text { Full flow } \\
\left(H_{j} \geqslant H_{j}^{\text {des }}\right)\end{array}$ \\
\hline $\begin{array}{l}\text { Wagner et al. (1988) } \\
\text { Equations I }\end{array}$ & $\mathbf{Q}_{j}^{\mathrm{avl}}=0$ & $\frac{\mathbf{Q}_{j}^{\text {avl }}}{\mathbf{Q}_{j}^{\text {req }}}=\left(\frac{H_{j}-H_{j}^{\min }}{H_{j}^{\text {des }}-H_{j}^{\min }}\right)^{0.5}$ & $\mathbf{Q}_{j}^{\text {avl }}=\mathbf{Q}_{j}^{\text {req }}$ \\
\hline $\begin{array}{l}\text { Germanopoulos-Gupta-Bhave } \\
\text { (GGB) (Gupta and Bhave, I996) } \\
\text { Equations } 2\end{array}$ & $\mathbf{Q}_{j}^{\mathrm{avl}}=0$ & $\frac{\mathbf{Q}_{j}^{\text {avl }}}{\mathbf{Q}_{j}^{\text {req }}}=1-10^{-5\left(\frac{H_{j}-H_{j}^{\min }}{H_{j}^{\text {req }}-H_{j}^{\min }}\right)}$ & $\mathbf{Q}_{j}^{\text {avl }}=\mathbf{Q}_{j}^{\text {req }}$ \\
\hline $\begin{array}{l}\text { Fujiwara and Ganesharajah (1993) } \\
\text { Equations } 3\end{array}$ & $\mathbf{Q}_{j}^{\text {avl }}=0$ & $\frac{\mathbf{Q}_{j}^{\text {avl }}}{\mathbf{Q}_{j}^{\text {req }}}=\frac{\int_{H_{j}^{\text {min }}}^{T_{j}}\left(H_{j}-H_{j}^{\text {min }}\right)\left(H_{j}^{\text {des }}-H_{j}^{\text {min }}\right) \mathrm{d} H}{\int_{H_{j}^{\min }}^{H_{j}^{\text {des }}}\left(H_{j}-H_{j}^{\text {min }}\right)\left(H_{j}^{\text {des }}-H_{j}^{\text {min }}\right) \mathrm{d} H}$ & $\mathbf{Q}_{j}^{\text {avl }}=\mathbf{Q}_{j}^{\text {req }}$ \\
\hline
\end{tabular}

$H_{\mathrm{j}}$, piezometric head at node $j ; H_{j}^{\min }$, piezometric head at node $j$ below which outflow is zero; $H_{j}^{\text {des }}$, piezometric head at node $j$ above which the demand is satisfied in full; $\mathbf{Q}_{j}^{\text {avl }}$, actual flow at node $j ; \mathbf{Q}_{j}^{\text {req }}$, demand at node $j$. 
modelling a demand node corresponding to a single tap. They proposed a relationship similar to Figure 1 on the basis that the flow at a node of the mathematical model would represent the abstraction at many demand points of the real WDS with different elevations. Furthermore, using HDA, Gupta and Bhave (1996) calculated the total flow supplied by a pipeline with several outlets for a range of pressures. Figure 1 would appear to be a reasonable approximation to the curve of actual pressure plotted against flow for the pipeline as a whole.

Udo and Ozawa (2001) proposed the following PDDF

$\left.\begin{array}{|l|}\boldsymbol{Q}_{j}^{\text {avl }}=0 \text { if }\left(H_{j}-E_{j}\right) \leqslant 0 \\ \boldsymbol{Q}_{j}^{\text {avl }}=0.0189 \boldsymbol{Q}_{j}^{\mathrm{req}}\left(H_{j}-E_{j}\right)^{2} \\ \text { if } 0<\left(H_{j}-E_{j}\right) \leqslant 6.4176 \mathrm{~m} \\ \boldsymbol{Q}_{j}^{\text {avl }}=\boldsymbol{Q}_{j}^{\mathrm{req}}\left\{\frac{\tan ^{-1}\left[1 \cdot 3\left(H_{j}-E_{j}-9 \cdot 5\right)\right]}{\pi}+0.5\right\} \\ \text { if } 6.4176 \mathrm{~m}<\left(H_{j}-E_{j}\right) \leqslant 12.582 \mathrm{~m} \\ \boldsymbol{Q}_{j}^{\text {avl }}=\boldsymbol{Q}_{j}^{\mathrm{req}}\left[1-0.0189\left(H_{j}-E_{j}-19\right)^{2}\right] \\ \text { if } 12.582 \mathrm{~m}<\left(H_{j}-E_{j}\right) \leqslant 19.0 \mathrm{~m} \\ \boldsymbol{Q}_{j}^{\text {avl }}=\boldsymbol{Q}_{j}^{\mathrm{req}} \text { if }\left(H_{j}-E_{j}\right)>19.0 \mathrm{~m}\end{array}\right\}$

where $E_{j}$ is the elevation of node $j$; $H_{j}$ is the piezometric head; $\boldsymbol{Q}_{j}^{\text {avl }}$ is the actual flow; $\boldsymbol{Q}_{j}^{\text {req }}$ is the demand; and $H_{j}-E_{j}$ is the pressure head.

Cullinane et al. (1992) presented a curve based on the cumulative normal distribution function - that is

\begin{tabular}{|l|l|}
\hline 5 & $Q_{j}^{\text {avl }}=\frac{Q_{j}^{\text {req }}}{\sqrt{2 \pi}} \int_{-\infty}^{\left(H_{j}-E_{j}-\mu_{j}\right) / \sigma_{j}} \exp \left(-t^{5 / 2}\right) \mathrm{d} t$ \\
\hline
\end{tabular}

in which $\mu_{j}$ and $\sigma_{j}$ are, respectively, the mean value and standard deviation of $H_{j}-E_{j}$. Equation 5 is evaluated numerically as it has no analytical form; although polynomial approximations of the cumulative normal distribution function may be used, it is worth noting that values of $\mu_{j}$ and $\sigma_{j}$ are not readily available.

The new PDDF proposed is

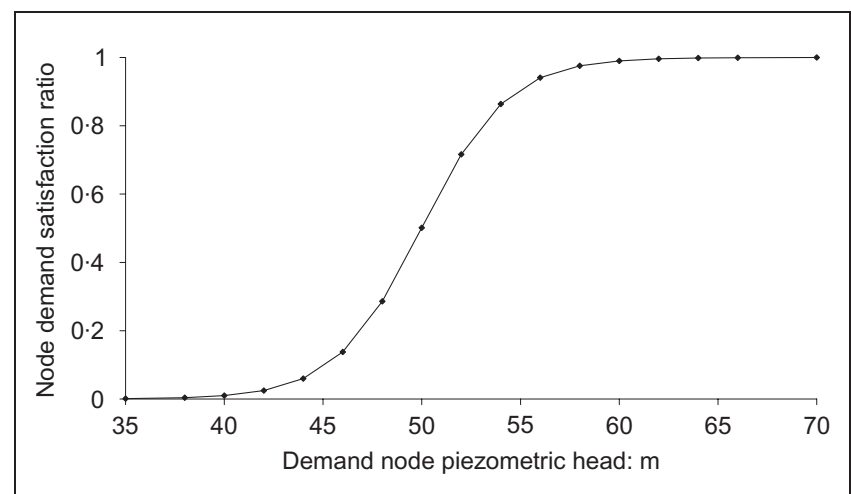

Figure I. Proposed pressure-dependent demand function

$$
\boldsymbol{Q}_{j}\left(H_{j}\right)=\boldsymbol{Q}_{j}^{\mathrm{req}} \frac{\exp \left(\alpha_{j}+\beta_{j} H_{j}\right)}{1+\exp \left(\alpha_{j}+\beta_{j} H_{j}\right)}
$$

where $\boldsymbol{Q}_{j}$ and $H_{j}$ are the flow and piezometric head, respectively, at node $j$. The values of the parameters $\alpha_{j}$ and $\beta_{j}$ are determined using relevant field data for the node in question. $\boldsymbol{Q}_{j}$ req is the demand at node $j$. When a node has a low pressure, it may supply only a small proportion of the demand or there may be no flow at all. By contrast, if sufficient pressure is available, the flow is equal to the demand. Equation 6 approximates the performance of a WDS under both normal and pressure-deficient conditions given the arguments $\alpha_{j}$ and $\beta_{j}$.

System-specific data are required to ascertain the values of $\alpha$ and $\beta$ for any network. In the absence of field data, default expressions may be obtained as follows. Taking $\boldsymbol{Q}_{j}\left(H_{j}^{\text {des }}\right)=0.999 \boldsymbol{Q}_{j}^{\text {req }}$ and $\boldsymbol{Q}_{j}\left(H_{j}^{\text {min }}\right)=0.01 \boldsymbol{Q}_{j}^{\text {req }}$, say, Equation 6 gives two simultaneous equations whose solution is

\begin{tabular}{|l|l|}
\hline 7 & $\alpha_{j}=\frac{-4.595 H_{j}^{\mathrm{des}}-6.907 H_{j}^{\min }}{H_{\mathrm{j}}^{\mathrm{des}}-H_{j}^{\min }}$ \\
\hline
\end{tabular}

$$
\beta_{j}=\frac{11 \cdot 502}{H_{j}^{\mathrm{des}}-H_{j}^{\min }}
$$

in which $H_{j}^{\text {des }}$ is the piezometric head above which $\boldsymbol{Q}_{j}=\boldsymbol{Q}_{j}^{\text {req }}$ and $H_{j}^{\text {min }}$ is the piezometric head below which $\boldsymbol{Q}_{j}=0$. For simplicity, $H_{j}^{\min }$ is often taken as the nodal elevation, $E_{j}$. However, a higher, more appropriate value can be used instead. The form of Equation 6 is shown in Figure 1. To the best of our knowledge, Equation 6 is the only PDDF which obviates the need for the extra conditions $\boldsymbol{Q}_{j}\left(H_{j} \leqslant H_{j}^{\mathrm{min}}\right)=0$ and $\boldsymbol{Q}_{j}\left(H_{j} \geqslant H_{j}^{\text {des }}\right)=\boldsymbol{Q}_{j}^{\text {req }}$. Equation 6 has the advantages of simplicity and ease of incorporation into the WDS constitutive equations. Furthermore, unlike other PDDFs, the derivative of Equation 6 has no discontinuities at $H_{j}=H_{j}^{\text {des }}$ and $H_{j}=H_{j}^{\min }$, which is an important factor in the computational solution of the system of equations. In effect, Equation 6 offers a seamless transition between normal and pressure-deficient operating conditions because it consists of a single continuous function that applies to all the three pressure regimes of $H_{j} \leqslant H_{j}^{\min }, H_{j}^{\min }<H_{j}<H_{j}^{\text {des }}$ and $H_{j} \geqslant H_{j}^{\text {des }}$ and has a continuous derivative.

\section{CONSTITUTIVE EQUATIONS}

The flow rate in a pipe may be related to the piezometric heads at the ends of the pipe by the Hazen-Williams equation as

9 $\boldsymbol{Q}_{i j}=R_{i j}\left(H_{i}-H_{j}\right)$

where $\boldsymbol{Q}_{i j}$ is the pipe flow rate, $H_{i}$ and $H_{j}$ are the piezometric heads at the nodes connected by the pipe. $R_{i j}$ incorporates the pipe resistance, namely 


$$
R_{i j}=\delta \frac{C_{i j} D_{i j}^{2 \cdot 63}}{L_{i j}^{0.54}\left|H_{i}-H_{j}\right|^{0.46}}
$$

where $\delta$ is a dimensionless factor whose value is $0 \cdot 2785$ in SI units, $C_{i j}$ is the Hazen-Williams roughness coefficient, $D_{i j}$ and $L_{i j}$ are the diameter and length, respectively. Equation 10 gives results that are accurate enough for the flow conditions commonly found in WDSs. The Darcy-Weisbach formula is more accurate as it involves a pipe friction factor whose value varies with the turbulence of the flow (unlike $C_{i j}$ which is assumed constant for a given pipe).

The formulation herein can handle multiple sources, pumps and valves. The equations for pumps and valves can be found in textbooks on WDSs. Some of the more common appurtenances are mentioned below. Non-return valves (NRVs) are used to ensure that water can flow in one direction through the pipeline but cannot flow in the opposite direction. A pipe fitted with an NRV is modelled as

$$
\text { I I } \boldsymbol{Q}_{i j}=\left\{\begin{array}{cc}
R_{i j} \operatorname{sign}\left(H_{i}-H_{j}\right)\left|H_{i}-H_{j}\right|^{0.54} & H_{i} \geqslant H_{j} \\
0 & H_{i}<H_{j}
\end{array}\right.
$$

where $\operatorname{sign}\left(H_{i}-H_{j}\right)=+1$ if $H_{i} \geqslant H_{j}$ and $\operatorname{sign}\left(H_{i}-H_{j}\right)=-1$ if $H_{i}<H_{j}$.

Pressure-reducing valves (PRVs) are designed to prevent the downstream pressure from exceeding a set value, and are used for example in situations where high downstream pressures are undesirable. A PRV is characterised in a model by the downstream pressure that it attempts to maintain and its control status. A PRV is modelled as

$$
\text { I2 } \boldsymbol{Q}_{i j}= \begin{cases}R_{i j}\left|H_{\text {set }}-H_{j}\right|^{0.54} & H_{i} \geqslant H_{\text {set }} \geqslant H_{j} \\ R_{i j} \operatorname{sign}\left(H_{i}-H_{j}\right)\left|H_{i}-H_{j}\right|^{0.54} & H_{\text {set }}>H_{i}>H_{j} \\ 0 & H_{\text {set }}<H_{j}\end{cases}
$$

where $H_{\text {set }}$ is the required piezometric head (pressure setting) downstream of the PRV.

A pump is typically modelled using a function such as

$$
h_{\mathrm{p}}=H_{j}-H_{i}=h_{0}-c Q_{\mathrm{p}}^{m}
$$

where $h_{\mathrm{p}}$ is the increase in head due to the pump, $h_{0}$ is the cutoff head, $\boldsymbol{Q}_{\mathrm{p}}$ is the discharge while c and $m$ are coefficients. The discharge is thus expressed as

I4 $\quad \boldsymbol{Q}_{\mathrm{p}}=\boldsymbol{Q}_{i j}=\left[\frac{h_{0}-\left(H_{j}-H_{i}\right)}{c}\right]^{1 / m} \quad \boldsymbol{Q}_{i j} \geqslant 0$

A pressure-sustaining valve (PSV) prevents the upstream pressure from dropping below a set value. The equation for a PSV can be written as follows
I5 $\quad \boldsymbol{Q}_{i j}=\left\{\begin{array}{cc}R_{i j}\left|H_{i}-H_{\mathrm{psv}}\right|^{0.54} & H_{i} \geqslant H_{\mathrm{psv}} \geqslant H_{j} \\ R_{i j}\left|H_{i}-H_{j}\right|^{0.54} & H_{i}>H_{j}>H_{\mathrm{psv}} \\ 0 & H_{i}<H_{\mathrm{psv}}\end{array}\right.$

where $H_{\mathrm{psv}}$ is the pressure setting.

To set up the constitutive equations, the flow continuity equation can be expressed for each node, $i$, as

$$
F_{i}\left(H_{i}, H_{j}\right)=\sum_{j \in N_{i}} \boldsymbol{Q}_{i j}-\boldsymbol{Q}_{i}\left(H_{i}\right)=0
$$

where $\boldsymbol{Q}_{i j}$ is the flow in link $i j$ (i.e. pipe, pump or valve), $\boldsymbol{Q}_{i}\left(H_{i}\right)$ is the head-dependent nodal flow and $N_{i}$ represents all the nodes connected to node $i$.

An outline of the numerical procedure developed to solve Equation 16 is presented in the following algorithm.

Step 1 . Set $k=0$. Set initial heads $H^{k} ; k$ represents the iteration number.

Step 2. Compute $F\left(H^{k}\right)$. If $\left\|F\left(H^{k}\right)\right\| \leqslant \varepsilon$, where $\varepsilon$ is a predefined tolerance, the algorithm terminates with $H^{k}$ as the solution. Otherwise, continue.

Step 3. Compute the Jacobian $J\left(H^{k}\right)$.

Step 4. Solve $J\left(H^{k}\right) \delta H^{k+1}=-F\left(H^{k}\right)$ for the change in nodal heads $\delta H^{k+1}$.

Step 5. Compute the value of the relaxation coefficient $\lambda^{k+1}$ (a scalar) to minimise $\left\|F\left(H^{k}+\lambda^{k+1} \delta H^{k+1}\right)\right\|$ with respect to $\lambda^{k+1}$; $0<\lambda^{k+1} \leqslant 1 \cdot 0$.

Step 6. Set $H^{k+1}=H^{k}+\lambda^{k+1} \delta H^{k+1}$ and go to Step 2 .

The formulation in Equation 16 is based on the unknown nodal piezometric heads, $\mathrm{H}$. The solution procedure developed in this research uses a Newton-Raphson scheme with line searches and backtracking in each iteration. The Newton step $\delta H=-J^{-1} . F$ provides a descent direction for the norm $|F|^{2}$. Line searches and backtracking along the $\delta H$ direction help ensure that successive iterates reduce the merit function $|F|^{2}$. Other criteria are used to guard against the merit function decreasing too slowly and/or excessively small steps being taken. For example, in each iteration the full Newton step $\delta H$ should be tried first to take full advantage of its quadratic convergence near the solution. However, $\delta H$ is not allowed to exceed a specified maximum step length to help ensure that $F$ is not evaluated in regions within which it may be undefined. The solution strategy outlined above is robust; it converges to the solution from almost any starting point (Press et al., 1992). The authors' prototype FORTRAN 90 implementation of the algorithm is called PRAAWDS (Program for the Realistic Analysis of the Availability of Water in Distribution Systems). Some of its features are given below.

Extensive testing has shown that the method is robust, globally convergent and offers seamless and realistic simulations for all pressure regimes without operator intervention. There is a choice of four PDDFs (Equations 1, 2, 3 and 6) including an option to choose DDA instead of HDA in the computer program 
with an integrated procedure for setting the initial piezometric heads $H^{k=0}$ without operator intervention. Pumps and valves (NRVs, PRVs, PSVs and flow control valves) can be handled and pipes can be declared as open or closed. The program is easy to use and expertise beyond the basics of WDSs is not required. Extra data are not required either. However, this may change with future sophistication. An innovative post-analysis technique for verifying HDA results is included as an option; nodal flows from WDSs with insufficient pressure will be different from the demands. If the HDA nodal flows are correct, then using the HDA flows as a new set of demands under the same conditions should yield DDA nodal heads and pipe flows that are identical to the original HDA heads and pipe flows. This new test is simple, but it is extremely efficient, powerful and effective as demonstrated in Ackley et al. (2001), for example.

\section{EXAMPLES, RESULTS AND DISCUSSION}

This section contains results for three examples including HDA and DDA obtained with PRAAWDS on a Pentium III $800 \mathrm{MHz}$ PC.

\section{I. Example I}

The first example is based on the simple network of Figure 2 (Fujiwara and Ganesharajah, 1993). $H_{j}^{\text {des }}=60 \mathrm{~m}$ for all demand nodes; $H_{j}^{\mathrm{min}}=45 \mathrm{~m}$ for nodes 3 and $4,50 \mathrm{~m}$ for nodes 1 and 2 and $55 \mathrm{~m}$ for nodes 5 and 6 . Source head values between 90 and $42 \mathrm{~m}$ were used. The results are summarised in Figure 3, which shows that the proposed PDDF needed fewer iterations. Figure 4 shows the hydraulic performance of the network based on the new PDDF, which can be compared with Figure 5 (Ackley et al., 2001; Tanyimboh et al., 2003) that is based on the Wagner et al. (1988) function.

The network performances under low-pressure conditions as shown in Figure 4 suggest that each node may have a unique head-flow curve. It is worth observing that the nodal

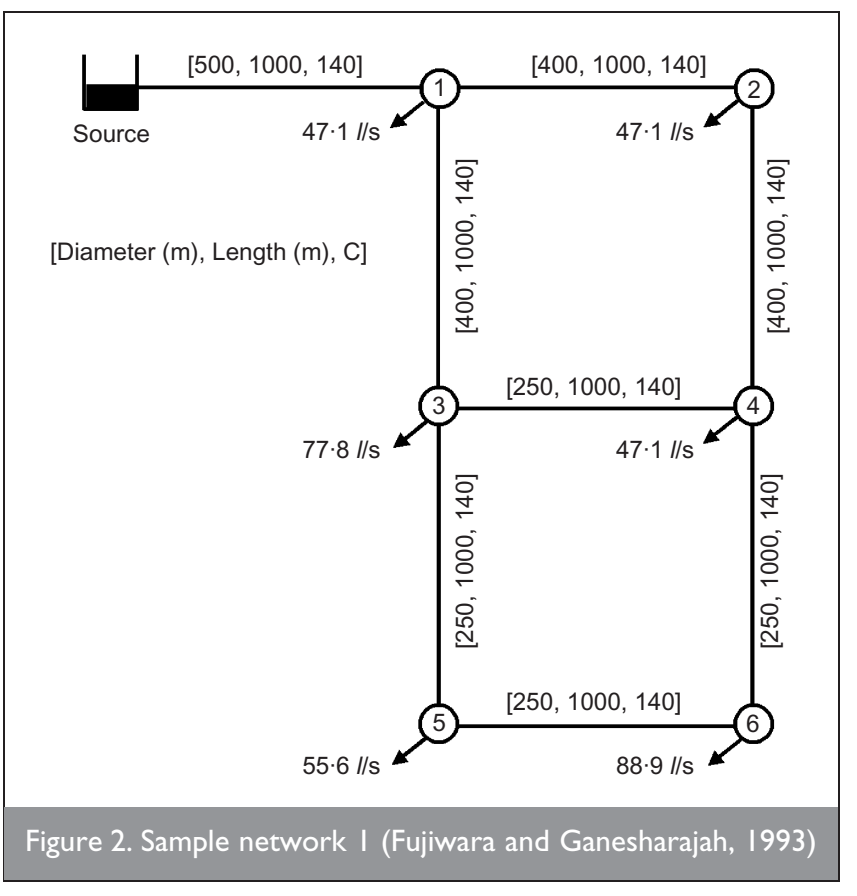

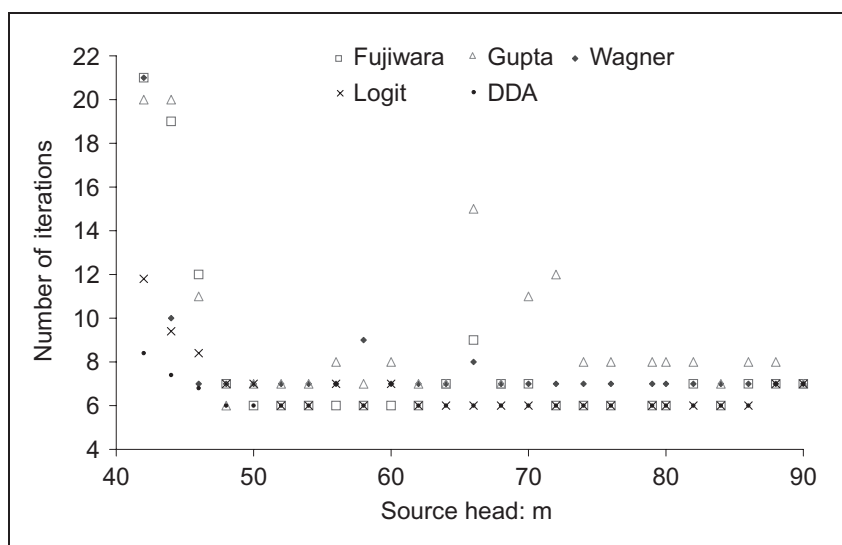

Figure 3. Number of iterations to achieve convergence for network I
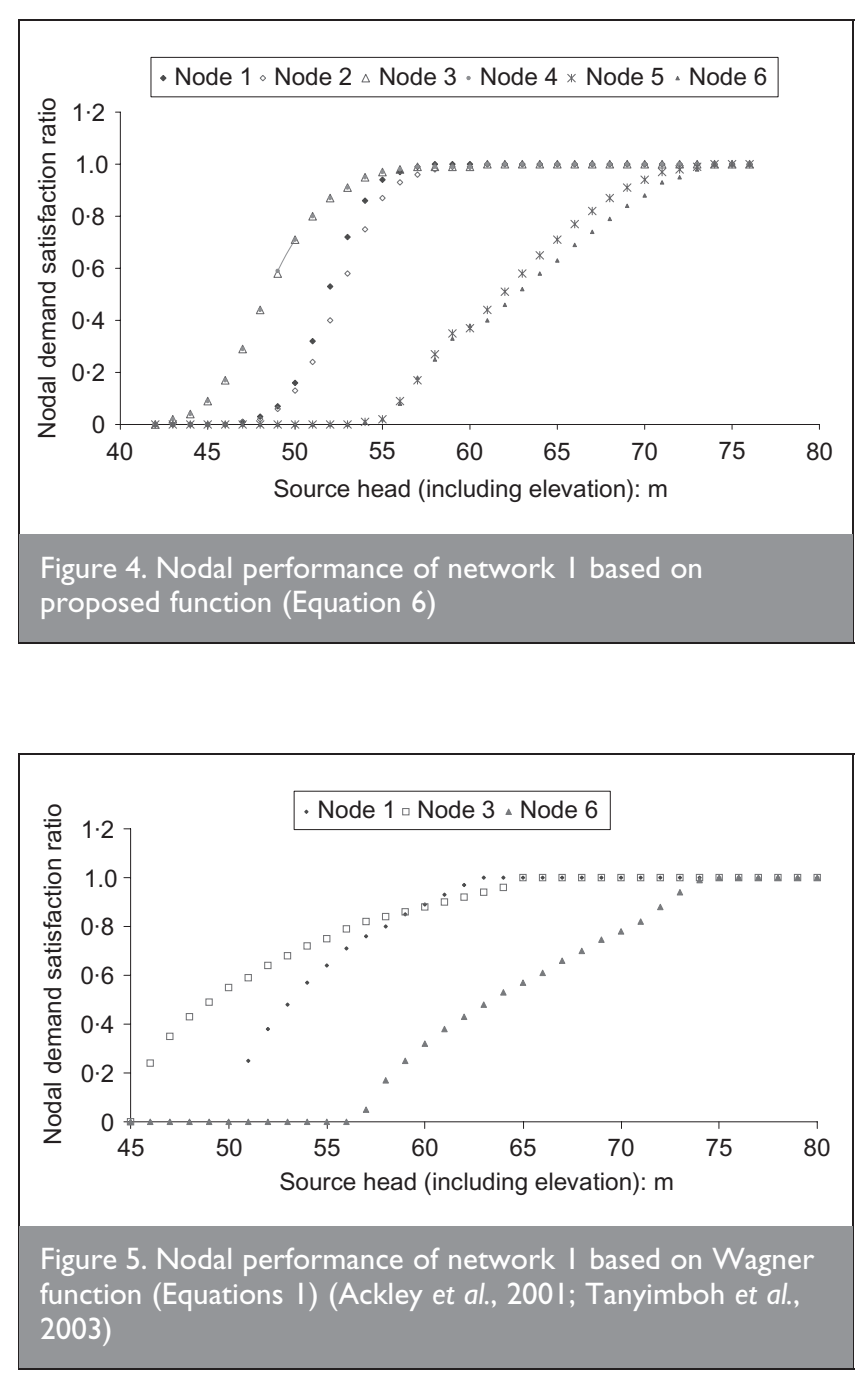

performances reflect: $(a)$ the minimum piezometric head values; $(b)$ the distances from the source; and (c) the magnitudes of the demands. For example, compare nodes 1 and 2 with nodes 3 and 4 . Although it is expected that different PDDFs will result in differences in the predictions of nodal flows, Figure 5 would appear to suggest that the various PDDFs may have different sensitivities to the relevant parameters. This is illustrated by the curves for nodes 1 and 3 in Figures 4 and 5. It may be noted that $H_{j}^{\min }$ is $45 \mathrm{~m}$ for node 3 and $50 \mathrm{~m}$ for node 1 . 


\subsection{Example 2}

The second example is based on a WDS from Reddy and Elango (1989). The network contains 70 pipes, 36 demand nodes and one source node (Figure 6). The pipe data and the elevations of the nodes are reported in Reddy and Elango (1989). The effective head at the source was varied from 10 to $25 \mathrm{~m}$. The nodal demands are all 80 litres/min while the residual heads for full and zero flow are 6.17 and $0 \mathrm{~m}$, respectively. The results are summarised in Figures 7 and 8. The CPU time was approximately $0.03 \mathrm{~s}$ for each simulation. It should be noted that unlike the other parts of this paper, the nodal heads in this example are pressure heads, because the values of the nodal elevations have been omitted for brevity. It is also worth noting that the DDA and logit function simulations required a comparable number of iterations down to a source head of about $19 \mathrm{~m}$ after which the logit function performed better (Figure 7).

\subsection{Example 3}

The third example is based on Jeppson and Davis (1976). The pipe data and demand at each node are given in Figure 9. The hydraulic characteristics of the pumps were represented by $h_{\mathrm{p}}=26.67-1042 Q^{2}$ and $h_{\mathrm{p}}=33.33-1029 Q^{2}$ for $\mathrm{P} 10$ and P11, respectively. The setting for the PRV was $140 \mathrm{~m}$. The head at supply node 10 was decreased from 250 to $100 \mathrm{~m}$ while the head at node 11 was maintained at a constant value of $180 \mathrm{~m}$. Additional data for the nodes are shown in Table 2. Figure 10 shows the performance of the WDS for the proposed PDDF and Figures 11(a) and (b) show the performance of the solution algorithm. Figures 11(a) and (b) show that the logit function generally requires fewer iterations.

\subsection{Comparison of pressure-dependent demand functions}

Table 3 shows a comparison of the PDDFs. Based on the examples considered here, the proposed function compares

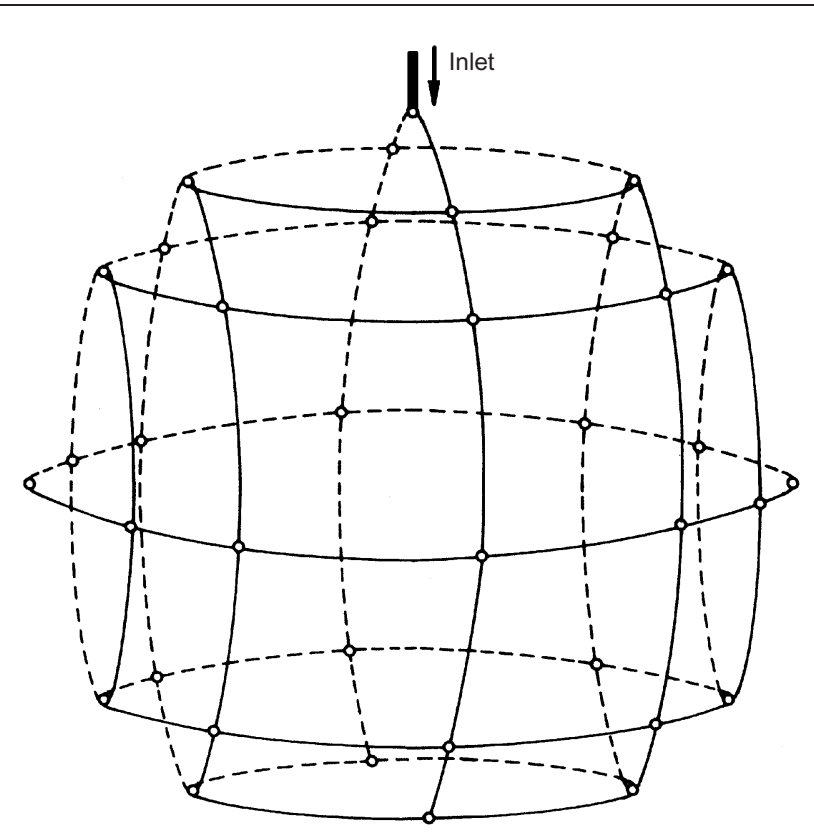

Figure 6. Sample network 2

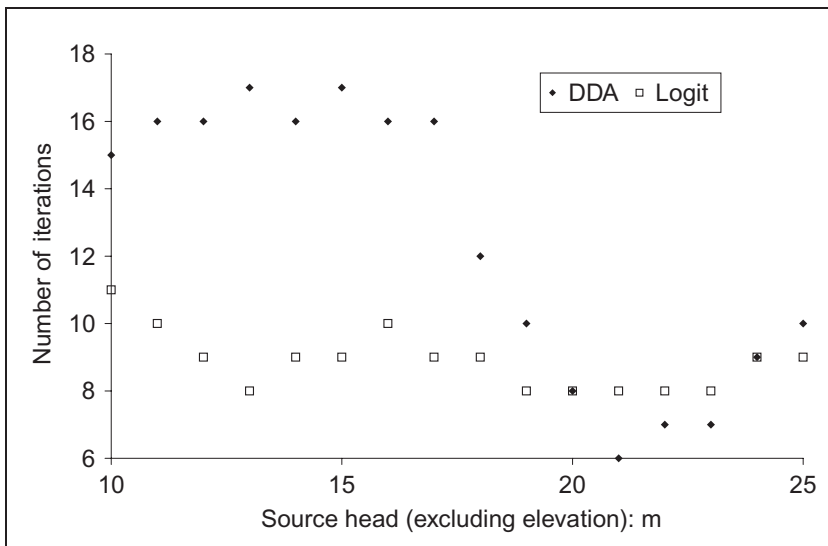

Figure 7. Computational performance of proposed function on network 2

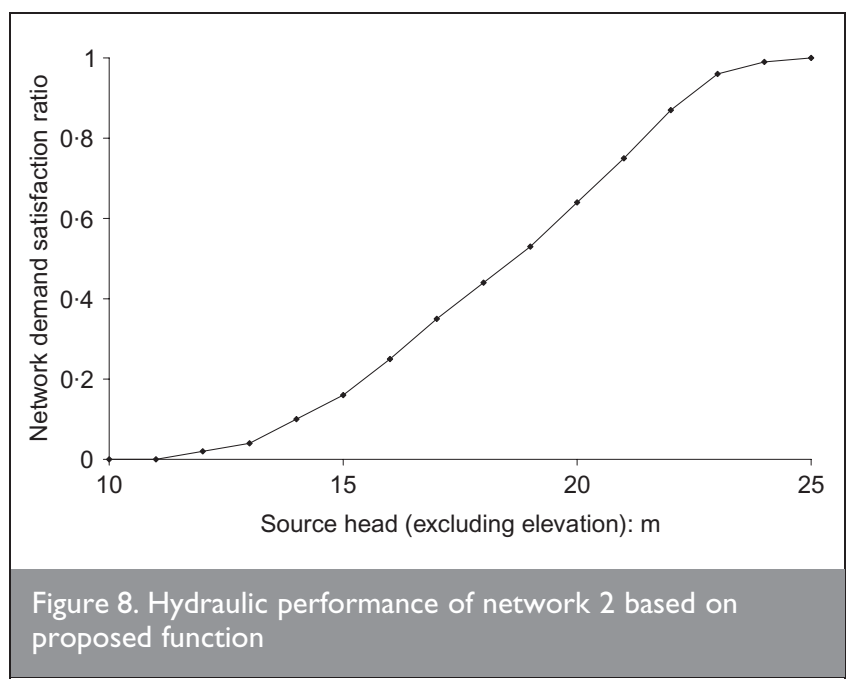

favourably against both DDA and other PDDFs. These results would appear to highlight the computational difficulties associated with discontinuous functions. For example, Figure 2, which corresponds to sample network 1, shows several unexpectedly high values of the number of iterations for all the PDDFs except for the proposed function and DDA. It should be noted that DSRs for nodes or networks have not been presented herein for DDA. The reason is that the DSR for DDA is always $100 \%$ regardless of whether this implied 100\% satisfaction ratio is achievable or not.

\section{CONCLUSIONS}

A new pressure-dependent demand function (PDDF) for WDSs has been proposed. It consists of a single function which covers the entire range from zero through to full flow. A major advantage of both the function and its derivative is that they do not have discontinuities. The new PDDF is ideal for incorporation in the WDS system of equations. The method developed to solve the system of equations employs a robust Newton-Raphson procedure. Evidence of the robustness includes the ability of the computer program (PRAAWDS) to run smoothly and produce realistic results, even at extremely low network-wide demand satisfaction levels. The program has a routine for selecting the initial 
Diameter $(\mathrm{mm})$, length $(\mathrm{m}), \mathrm{C}]$

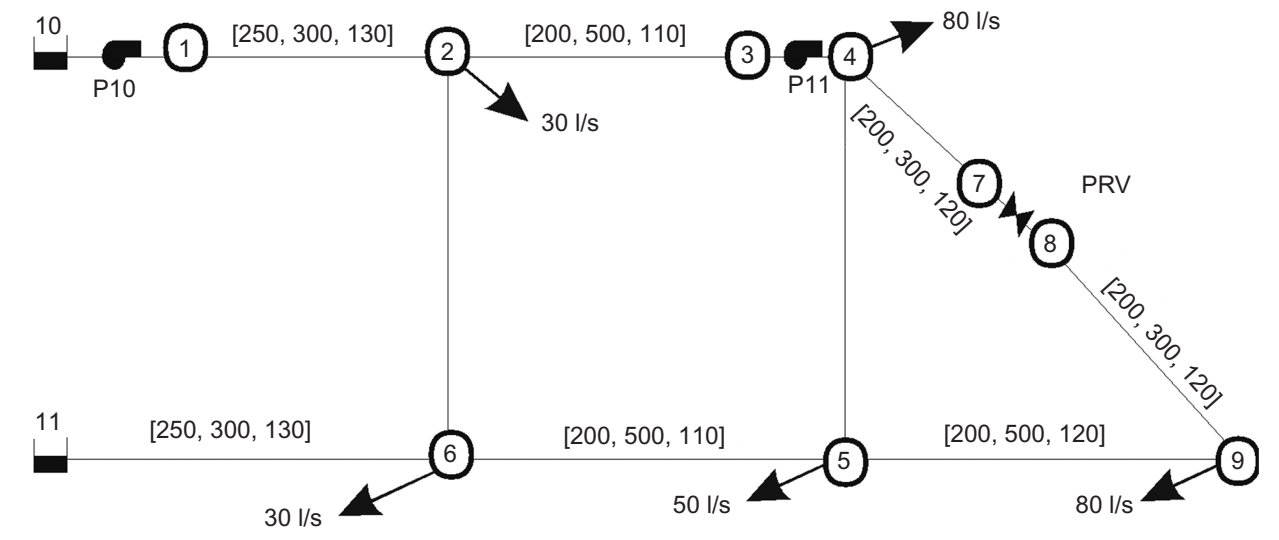

Figure 9. Sample network 3

\begin{tabular}{llllll} 
Nodes & $1-2$ & 3 & $4-5$ & 6 & $7-9$ \\
\hline $\begin{array}{l}\text { Minimum piezometric head: } m \\
\text { Desired piezometric head: } m\end{array}$ & 150 & 100 & 130 & 150 & 120 \\
& 170 & 120 & 150 & 170 & 140 \\
Table 2. Node data for network 3 & & & & & \\
\end{tabular}
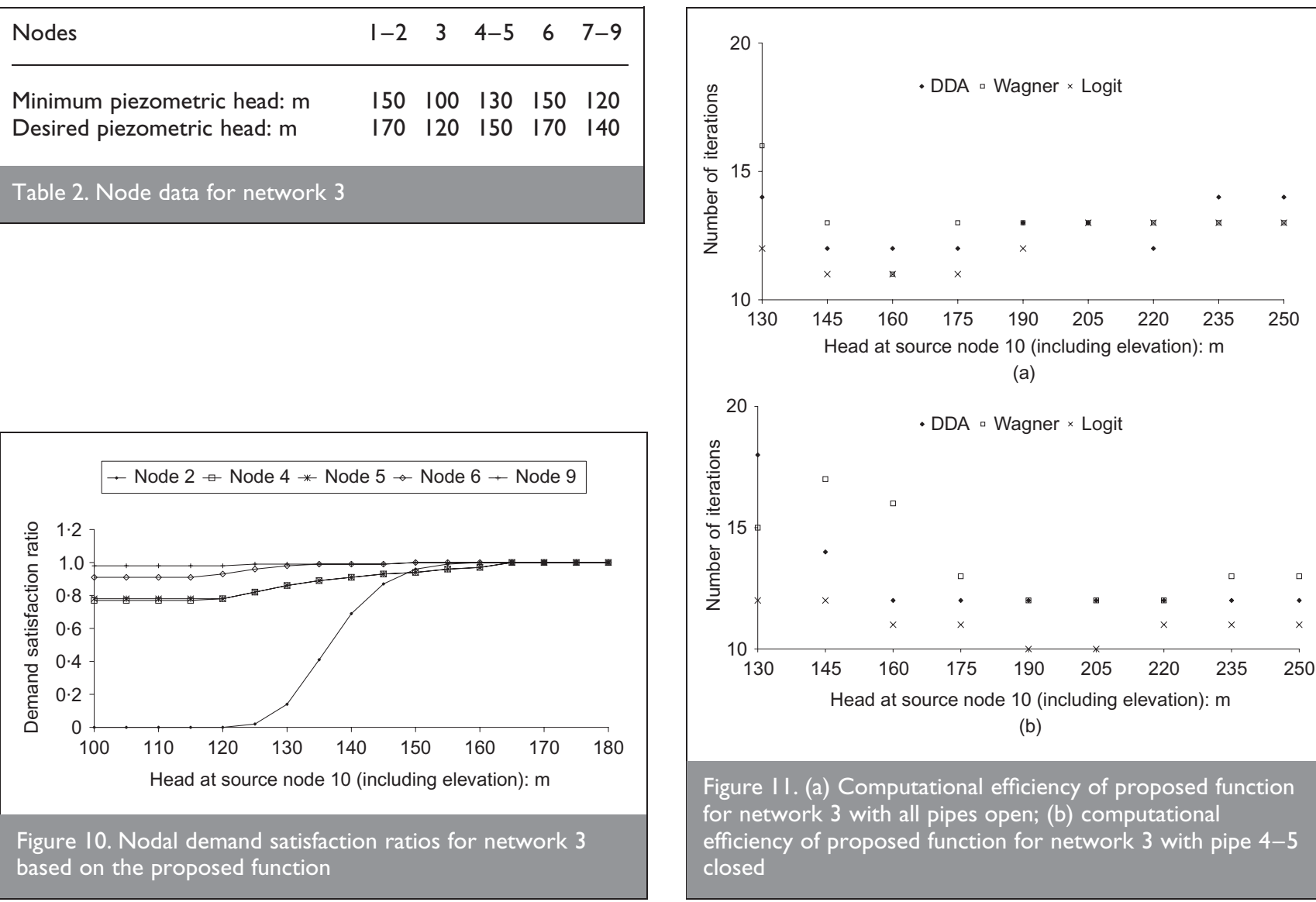

(a)

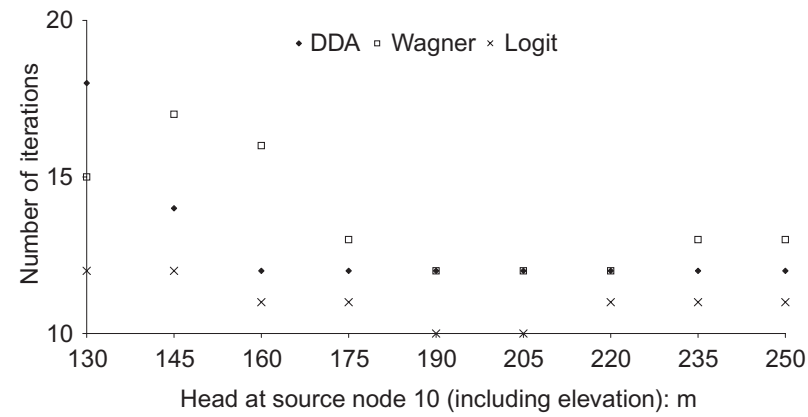

(b)

Figure II. (a) Computational efficiency of proposed function for network 3 with all pipes open; (b) computational

efficiency of proposed function for network 3 with pipe 4-5 closed

\begin{tabular}{|lrrrrr|}
\hline & DDA & Logit & Wagner & GGB* & Fujiwara \\
\hline Network 1 & 6.7 & 7.0 & 7.5 & 9.2 & 7.8 \\
Network 2 & 12.4 & 8.8 & 8.7 & 8.9 & 9.1 \\
Network 3 (pipe 4-5 open) & 12.8 & 12.1 & 12.0 & 11.0 & 17.0 \\
Network 3 (pipe 4-5 closed) & 12.8 & 11.0 & 15.8 & 16.2 & 17.6 \\
\hline * Germanopoulos-Gupta-Bhave & \multicolumn{7}{c}{} \\
\hline Table 3. Average number of iterations per simulation (for range of source heads) & \\
\hline
\end{tabular}


heads and in general multiple trials are not required. It can handle pumps and several types of valve, simulate both normal and pressure-deficient operating conditions seamlessly and has four PDDFs. Finally, extensive testing based on PRAAWDS including the examples herein suggests that, in general, HDA simulations do not necessarily take longer than DDA.

\section{REFERENCES}

Ackley JRL, Tanyimboh TT, Tahar B and Templeman AB (2001) Head-driven analysis of water distribution systems. In Water Software Systems: Theory and Applications (Ulanicki B, Coulbeck B and Rance J (eds)). Research Studies Press, Taunton, UK, Vol. 1, pp. 183-192.

Ang WK and Jowitt PW (2006) Solution for water distribution systems under pressure-deficient conditions. Journal of Water Resources Planning and Management 132(3): 175182.

Bhave PR (1991) Analysis of Flow in Water Distribution Networks. Technomic Publishing, Lancaster, UK.

Chandapillai J (1991) Realistic simulation of water distribution system. Journal of Transportation Engineering 117(2): 258263.

Cullinane MJ, Lansey KE and Mays LW (1992) Optimisationavailability-based design of water-distribution networks. Journal of Hydraulic Engineering 118(3): 420-441.

Fujiwara 0 and Ganesharajah T (1993) Reliability assessment of water supply systems with storage and distribution networks. Water Resources Research 29(8): 2917-2924.

Germanopoulos G (1985) A technical note on the inclusion of pressure dependent demand and leakage terms in water supply network models. Civil Engineering Systems 2(3): $171-179$.

Giustolisi 0, Kapelan Z and Savic D (2008) Extended period simulation analysis considering valve shutdowns. Journal of Water Resources Planning and Management 134(6): 527 537.

Gupta R and Bhave PR (1996) Comparison of methods for predicting deficient network performance. Journal of Water Resources Planning and Management 122(3): 214-217.

Gupta R, Sawarkar VR and Bhave PR (2003) Application of Newton-Raphson method in optimal design of water distribution networks. Journal of Indian Water Works Association 35(1): 31-37.
Jeppson RW and Davis A (1976) Pressure reducing valves in pipe network analyses. Journal of Hydraulics Division 102(HY7): 987-1001.

Kalungi P and Tanyimboh TT (2003) Redundancy model for water distribution systems. Reliability Engineering and System Safety 82(3): 275-286.

Press WH, Teukolsky SA, Vetterling WT and Flannery BP (1992) Numerical Recipes in FORTRAN: The Art of Scientific Computing. Cambridge University Press, New York, USA.

Reddy LS and Elango K (1989) Analysis of water distribution networks with head dependent outlets. Civil Engineering Systems 6(3): 102-110.

Rossman LA (2007) Discussion of 'Solution for water distribution systems under pressure-deficient conditions'. Journal of Water Resources Planning and Management 133(6): 566-567.

Siew C and Tanyimboh TT (2009) Augmented gradient method for head dependent modelling of water distribution networks. Proceedings of the World Environmental and Water Resources Congress, Kansas City. ASCE, Reston, USA.

Tabesh M, Tanyimboh TT and Burrows R (2002) Head-driven simulation of water supply networks. International Journal of Engineering 15(1): 11-22.

Tanyimboh TT, Burd R, Burrows R and Tabesh M (1999) Modelling and reliability analysis of water distribution systems. Water Science and Technology, IAWQ 39(4): 249255.

Tanyimboh TT, Tahar B and Templeman AB (2003) Pressuredriven modelling of water distribution systems. Water Science and Technology - Water Supply 3(1-2): 255-262.

Todini E and Pilati S (1988) A gradient algorithm for the analysis of pipe networks. Computer Applications in Water Supply, Volume 1 (Coulbeck B and Orr C-H (eds)). Research Studies Press, Taunton, UK.

Udo A and Ozawa T (2001) Steady-state flow analysis of pipe networks considering reduction of flow in the case of low water pressures. Water Software Systems: Theory and Applications (Ulanicki B, Coulbeck B and Rance J (eds)). Research Studies Press, Taunton, UK, Vol. 1, pp. 73-182.

Wagner JM, Shamir U and Marks DH (1988) Water distribution reliability: simulation methods. Journal of Water Resources Planning and Management 114(3): 276-294.

Weisberg S (1985) Applied Linear Regression. Wiley, New York, USA.

\section{What do you think?}

To discuss this paper, please email up to 500 words to the editor at journals@ice.org.uk. Your contribution will be forwarded to the author(s) for a reply and, if considered appropriate by the editorial panel, will be published as discussion in a future issue of the journal.

Proceedings journals rely entirely on contributions sent in by civil engineering professionals, academics and students. Papers should be 2000-5000 words long (briefing papers should be 1000-2000 words long), with adequate illustrations and references. You can submit your paper online via www.icevirtuallibrary.com/content/journals, where you will also find detailed author guidelines. 